\title{
Primary Epiploic Appendagitis Mimicking Acute Abdomen in Emergency Department
}

\author{
Türker Acar ${ }^{1}$, Duran Efe ${ }^{2}$, Kazım Gemici ${ }^{3}$, Serkan Güneyli ${ }^{4}$ \\ 'Department of Radiology, Abant İzzet Baysal University, Training and Research Hospital, Bolu, Turkey \\ ${ }^{2}$ Department of Radiology, Mevlana University School of Medicine, Konya, Turkey \\ ${ }^{3}$ Department of Surgery, Mevlana University School of Medicine, Konya, Turkey \\ ${ }^{4}$ Department of Radiology, Bülent Ecevit University School of Medicine, Zonguldak, Turkey
}

A 27-year-old man presented to the emergency department of our institution with left lower quadrant pain that started $8 \mathrm{~h}$ ago. He did not describe kidney stone, chronic medical problem, or recent trauma in his anamnesis. His vital signs were stable, but physical examination revealed defense and rebond in the left lower quadrant. Laboratory findings were unremarkable except for mildly elevated C-reactive protein (CRP) levels of $6.28 \mathrm{mg} / \mathrm{L}$ (reference value: $0-5 \mathrm{mg} / \mathrm{L}$ ). He was referred to abdominal ultrasonography (USG) following laboratory tests and his written informed consent was obtained before imaging studies. Solid organs were in normal limits in USG, but a significantly increased echogenicity was noted in the pericolic fat tissue adjacent to the sigmoid colon surrounded by a hypoechoic border, which was suspicious for sigmoid diverticulitis (Figure 1).

Following USG, the patient was referred to computed tomography $(C T)$ with the preliminary diagnosis of sigmoid diverticulitis. CT showed a pericolic oval lesion of fat attenuation with a hyperattenuating ring and central dot sign in the same region that was diagnostic for epiploic appendagitis (EA); (Figure 2). However, no diverticula or diverticulitis was observed in the sigmoid colon.

EA is a rare and self-limited condition, mostly seen in middle-aged men, which is caused by either inflammation or ischemic changes related to torsion or venous infarction of the epiploic appendages (1). There are two forms of EA: primary and secondary. Primary EA results from spontaneous torsion of epiploic appendages, with resultant vascular occlusion and ischemia. In contrast, secondary EA is induced by the spread of adjacent inflammation from organs such as gallbladder, appendix, and colon (2).

Primary EA is a diagnostic challenge in emergency medicine; for example, right-sided primary EA is often confused with acute appen-

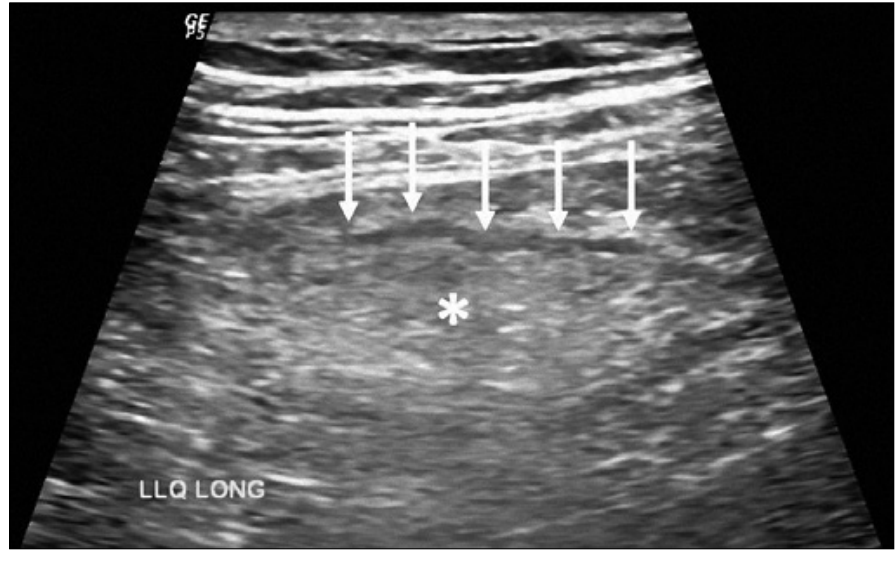

Figure 1. Longitudinal ultrasonographic image from left lower quadrant shows increased pericolic fat echogenicity (asterix) surrounded by a hypoechoic rim (arrows)

dicitis or right-sided diverticulitis, whereas left-sided primary EA is often misdiagnosed as sigmoid diverticulitis $(1,3)$.

EA is seen as a noncompressible hyperechoic small ovoid or round solid mass of adipose tissue between the colon and abdominal wall. The affected epiploic appendage, adherent to the colonic wall, is frequently found to be surrounded by a hypoechoic border during USG (1). CT is the most effective imaging method in EA diagnosis. A hyperattenuating ring sign that represents the inflamed visceral peritoneal covering of the epiploic appendage and central dot sign that corresponds to engorged or thrombosed central vessels or central areas of hemorrhage or fibrosis are the two major CT features of EA (1). In the present case, USG evaluation was first performed by a radiologist in the radiology department. Howev- 

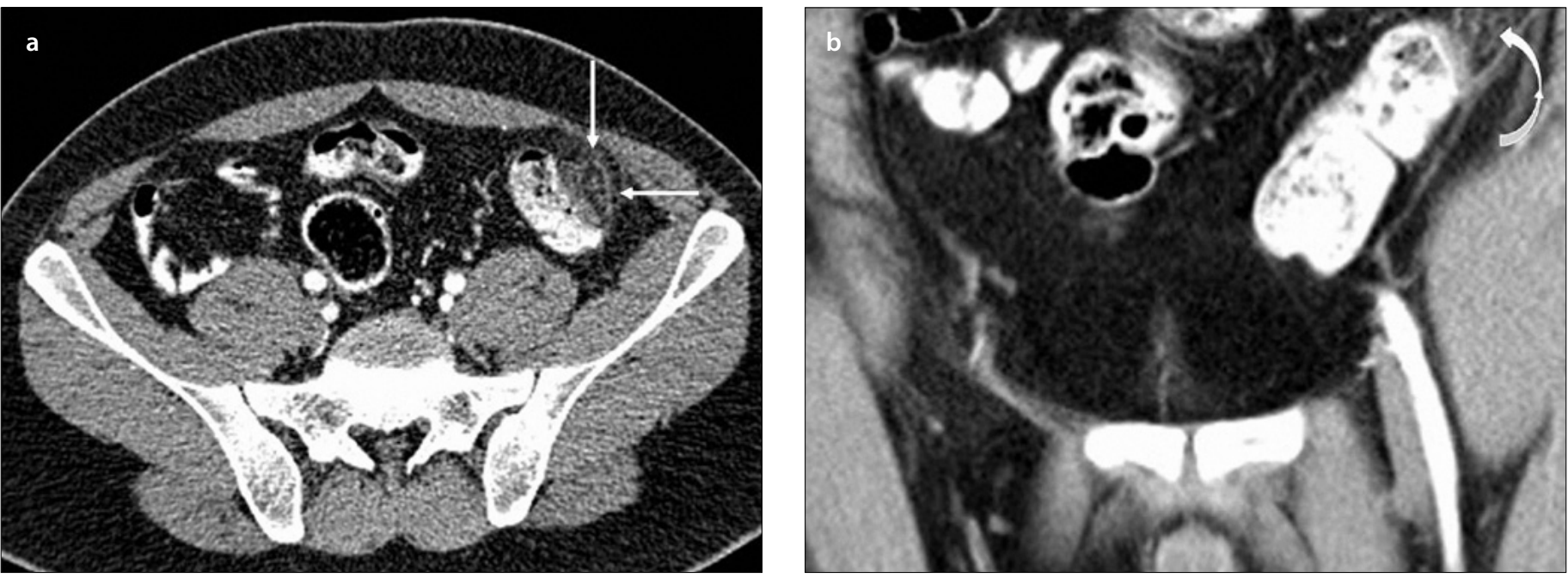

Figure 2. a, b. Axial (a) and coronal reconstruction (b) CT images show hyperattenuating ring sign (arrows) and central dot sign (curved arrow), diagnostic for epiploic appendagitis

er, our experience suggests that if clinicians were familiar with the sonographic features of EA, the same USG features could also be revealed in the emergency department, which would facilitate the diagnosis.

As EA is a self-limited condition, its treatment is conservative in nearly all cases and surgery is not recommended (3). The current patient was treated conservatively as mentioned in the literature, and after treatment his symptoms relieved and CRP level decreased.

In conclusion, EA is a self-limiting benign condition, which may mimic serious abdominal emergencies such as sigmoid diverticulitis. Because the management of EA is different, $\mathrm{CT}$ is a more reliable method for diagnosis.

Informed Consent: Written informed consent was obtained from patient who participated in this case.
Peer-review: Externally peer-reviewed.

Conflict of Interest: No conflict of interest was declared by the authors.

Financial Disclosure: The authors declared that this study has received no financial support.

\section{References}

1. Almeida AT, Melão L, Viamonte B, Cunha R, Pereira JM. Epiploic appendagitis: an entity frequently unknown to clinicians--diagnostic imaging, pitfalls, and look-alikes. Am J Roentgenol 2009; 193: 1243-51. [CrossRef]

2. Sorser SA, Maas LC, Yousif E, Maas L. Epiploic appendagitis: the great mimicker. South Med J 2009; 102: 1214-7. [CrossRef]

3. Sand M, Gelos M, Bechara FG, Sand D, Wiese TH, Steinstraesser L, et al. Epiploic appendagitis: clinical characteristics of an uncommon surgical diagnosis. BMC Surg 2007; 1: 7-11. [CrossRef] 